\title{
'ME DIERON ACÁ, ME ROMPIERON ALLÁ'. Políticas de relocalización de la población empobrecida en el barrio Coronel Dorrego, Santa Fe
}

\author{
Autora: Emilia Mosso \\ Universidad o Institución a la que pertenece: IIDUTyV/FAUD/UNMdP-CONICET \\ Directora de la tesis: Dra. Arq. Ana Núñez - IIDUTyV/FAUD/UNMdP \\ EMail:emiliamosso@gmail.com
}

\section{RESUMEN}

El objetivo de esta investigación es analizar las condiciones socio-habitacionales de las familias relocalizadas por los Programas Urbanos Integrales en el barrio Coronel Dorrego en la última década en la ciudad de Santa Fe. Preguntándonos ¿Cómo inciden las viviendas mínimas otorgadas por estos programas en el mejoramiento de las condiciones de vida de las familias relocalizadas? Para ello, a partir del análisis de las condiciones socio-habitacionales preexistentes a los procesos de relocalización, y las variaciones realizadas luego del traslado, investigamos la incidencia de la entrega de las viviendas en las condiciones de vida de las familias. Considerando que, mientras se generan ciertas mejorías en estas condiciones, se reproducen o perpetúan las condiciones estructurales de empobrecimiento de la población. En este marco, nos apoyamos en herramientas de tipo cuali-cuantitativo y en diversos instrumentos (análisis de documentos, entrevistas, trabajo de campo) y estrategias de abordaje (exploratoria y analítica).

Palabras clave: Empobrecimiento - políticas urbanas - condiciones de vida

\begin{abstract}
The objective of this research is to analyze the socio-residential conditions of the families relocated by the Comprehensive Urban Programs in the Coronel Dorrego neighborhood in the last decade in the city Santa Fe. Asking us, How do the minimal housing granted by these programs affect the improvement of the life conditions of the relocated families? For this, from the analysis of the pre-existing socio-housing conditions to the relocation processes, and the changes made after the transfer, we investigated the incidence of the delivery of the houses in the life conditions of the families. Considering that, in parallel with certain improvements under these conditions, the structural conditions of impoverishment of the population are reproduced or perpetuated. In this framework, we rely on qualitative-quantitative tools and various instruments (document analysis, interviews, field work) and strategies (exploratory and analytical).
\end{abstract}

Key words: Impoverishment - urban policies - life conditions 


\section{INTRODUCCIÓN}

Existe un amplio consenso en el campo urbano nutrido en justificar teóricamente a las políticas, los programas, los proyectos y a la propia normativa urbana, desde nociones y conceptos naturalizados. Entre estos se encuentran las nociones de vivienda digna, anclada a umbrales minimistas (Leguizamón, 2005) para la población empobrecida. Sin embargo, esta conceptualización, cuya génesis data desde las Campañas globales de la ONU y la CEPAL desde mediados de la década de 1970, es reproducida por intelectuales, instituciones locales e internacionales, organismos bilaterales de créditos, bancos mundiales y regionales de desarrollo, y por los propios gobiernos acríticamente década tras década.

Un claro ejemplo de la instrumentalización de este concepto, son los recientes Programas Urbanos Integrales -en adelante PUI-, que a la par de políticas de regularización dominial del suelo incluyen operatorias viviendas "sociales" y el mejoramiento o la dotación de infraestructuras, equipamientos y servicios del entorno barrial, entre otras cuestiones. Estos programas, financiados por organismos internacionales de crédito -en adelante Ol-, tienen entre sus argumentos teóricos la entrega de viviendas mínimas a familias empobrecidas incluyendo, en simultáneo, operatorias de relocalización de la población. Sin embargo, será desde la justificación de este supuesto hegemónico, entre otros, uno de los instrumentos que legitimizará, a nuestro entender, (re)ordenamientos espaciales de la población empobrecida: es decir, el otorgamiento de viviendas mínimas, dignas, sociales, módulos o soluciones habitacionales, entre otros, como instrumentos posibilitantes de desplazamientos de la población empobrecida.

En este sentido, este trabajo constituye un extracto de los resultados obtenidos a partir de una investigación propia más amplia realizada entre los años 2013 y $2017^{1}$, en donde buscamos analizar las condiciones socio-habitacionales de las familias relocalizadas por los PUI en el barrio Coronel Dorrego -en adelante CDen la ciudad de Santa Fe en la última década. Especialmente, nuestro análisis se basó en los instrumentos de política urbana que operan en estos programas, y en su incidencia en el mejoramiento de las condiciones de vida de la población involucrada; centrando nuestra atención en dos de ellos: las viviendas y las escrituras de domino otorgadas a las familias. En este marco, a partir del análisis de las condiciones socio-habitacionales preexistentes a los procesos de relocalización, y las variaciones realizadas luego del traslado, investigamos la incidencia de la entrega de las viviendas y las escrituras de dominio en las condiciones de vida de estas familias. Según nuestra comprensión, estos instrumentos de política, entre otros, mientras generarían ciertas mejorías en las condiciones de vida de la población, reproducirían las condiciones estructurales de empobrecimiento de la misma. La investigación realizada puso énfasis, en este marco, en dos aspectos entrelazados: uno de ellos fue el teórico, en donde indagamos en las interpelaciones ideológicas presentes en las políticas urbanas socio-habitacionales; y otro de ellos fue empírico, en donde analizamos cómo, cuándo y dónde estos supuestos son instrumentalizados en el espacio. Así, el presente trabajo expone algunas de las reflexiones obtenidas en este trabajo de campo y en los métodos e instrumentos para llevar adelante la investigación, a partir de las entrevistas realizadas a 23 familias relocalizadas por el PUI del Noreste en CD.

En este contexto, el análisis planteado resulta necesario dado que en la ciudad de Santa Fe hacia el año 2009, al menos 15.000 familias se encontraban en situaciones socio-habitacionales precarias de tenencia y otras 1000 estaban asentadas en áreas de riesgo hídrico ${ }^{2}$. Esta situación socio-habitacional se extendía en, al menos, 50 barrios localizados en el oeste, norte y el sector costero de la ciudad, y comprendía a una población de 70.000 habitantes. Es decir, implicaba un porcentaje cercano al $20 \%$ de la población santafesina residiendo en situaciones de tenencia precaria. Según datos actualizados, hacia finales del año 2017 en la ciudad se registraron 74 barrios en donde reside población en situaciones socio-habitacionales precarias, en los que viven 14.629 familias y comprende una cantidad aproximada de 48.288 personas ${ }^{3}$. Es decir, que a pesar de la instrumentalización de los programas y políticas urbanas en ese periodo ${ }^{4}$, se han incrementado la cantidad de sectores urbanos en donde residen familias en condiciones precarias de tenencia.

\footnotetext{
${ }^{1}$ Nos referimos a la tesis doctoral "INTERPELACIONES IDEOLÓGICAS SOBRE LA VIVIENDA. Políticas urbanas de ordenamiento espacial de la población empobrecida en Santa Fe (1985-2017)", investigación enmarcada tanto en la beca Doctoral de CONICET (2013/2018) como en el proyecto colectivo PICT 2013-2053, FONCyT/ANPCyT, ambos con la dirección de la Dra. Arq. Ana Núñez (IIDUTy Yv/FAUD, UNMdP/CONICET).

${ }^{2}$ Esta información fue obtenida a partir de la entrevista realizada en el año 2016 a la Directora de la Agencia Santa Fe Hábitat, Arq.Sara Lauría.

${ }^{3}$ Datos obtenidos del Relevamiento Nacional de Barrios Populares - RENABAP- realizado en forma conjunta por diferentes niveles de gobierno y organizaciones sociales. http://www.ellitoral.com/index.php/id um/165011-identifican-74-barrios-populares-en-la-ciudad-desanta-fe-relevamiento-del-gobierno-nacional-area-metropolitana.html Última visita 15/02/2018.

${ }^{4}$ Para una mayor profundización se recomienda ver Mosso,2017, op. Cit.
} 
LA CONCEPCIÓN DE LA INVESTIGACIÓN EMPIRICA

Para llevar a cabo la esta investigación, utilizamos métodos combinados de tipo cuali-cuantitativos, a partir de una estrategia de abordaje analítica apoyada en diversos instrumentos (análisis de documentos, entrevistas semi-estructuradas y en profundidad a informantes claves, trabajo de campo de tipo etnográfico, confección de mapeos y cartografía). Este encuadre metodológico amerita, sin embargo, algunas acotaciones.

\subsection{La unidad de análisis y sus fuentes}

Como unidad de análisis partimos de los programas urbanos integrales instrumentalizados desde el año 2012 en Santa Fe. Estos programas comprenden operatorias de relocalización de población, la construcción de módulos habitacionales para estas familias y su regularización dominial. En este marco, tomamos como caso empírico a las familias objeto de estos programas, las cuales han sido relocalizadas a nuevas viviendas entre los años 2013 y 2014 en el barrio CD. Más allá de este recorte temporal, indagamos a su vez en los desplazamientos previos de estas familias hasta llegar a su actual residencia. En este marco, buscamos analizar cómo estos procesos se relacionan con las condiciones de vida de la población, incluyendo su localización espacial, y cómo esta se relaciona con la configuración del orden urbano en general, a partir de sus desplazamientos previos. No obstante, para esta instancia y por las limitaciones de espacio pautadas, no profundizaremos en los desplazamientos espaciales investigados.

En este contexto, hemos trabajado con dos tipos de fuentes. Por un lado, nos propusimos realizar la indagación a través de la observación directa. En este marco, utilizamos fuentes primarias a partir del registro in situ. La técnica de relevamiento empírico que elegimos como principal instrumento de registro fue la realización de entrevistas semi-estructuradas que combinan preguntas prediseñadas y abiertas, considerándolas como preguntas disparadoras. Para ello, realizamos dos tipos de entrevistas: a) entrevistas en profundidad y conversaciones informales realizadas personalmente a informantes clave en el período 2013-2017, entre ellos, técnicos, funcionarios, referentes teóricos y barriales; y b) entrevistas en profundidad y conversaciones informales realizadas personalmente a 23 familias relocalizadas del barrio, sobre las cual profundizaremos en este trabajo.

En este contexto, comenzamos el trabajo de campo en el barrio CD a finales del año 2015, y retomamos las entrevistas a las familias entre los meses de abril a noviembre del año 2017, a fin de observar las transformaciones transcurridas en este proceso ${ }^{5}$. Las entrevistas las realizamos personalmente en el barrio, dentro de las viviendas de cada familia o bien en el ingreso de las mismas, y fueron hechas según el caso de manera individual o grupal. Las visitas al barrio las hicimos entre los días martes a jueves, entre la mañana y la siesta. En consonancia, también nos apoyamos en fuentes secundarias emitidas del ámbito internacional y local.

\subsection{Las etapas de la investigación y su profundización}

Estructuramos la investigación en tres etapas. Las dos primeras correspondieron a indagaciones teóricas en relación a los diferentes debates sobre la problemática habitacional, tanto en el ámbito internacional como local. Buscamos comprender, en ese sentido, el actual estado del arte sobre las conceptualizaciones de vivienda digna y tenencia segura, remitiéndonos al contexto histórico que data de la principios de la década de 1970, y fundamentalmente, en la incidencia en las últimas décadas de las campañas globales emergidas desde los organismos internacionales de financiamiento en esta línea -entre ellos la ONU, UN-Hábitat, CEPAL, BM, y los bancos regionales de desarrollo entre otros. No obstante, por motivos de espacio hemos omitido estas discusiones.

La tercera etapa, objeto de este trabajo, corresponde al caso empírico de investigación, el cual concierne a un proceso urbano reciente y en estadio inicial. Dos cuestiones incidieron en esta elección. Por un lado, la accesibilidad a información obtenida en cuanto a planimetrías y relevamientos sociales. Por otro, la disponibilidad de algunos contactos en el barrio para poder realizar las entrevistas con las familias y el trabajo de campo. De esta manera, la selección de la relocalización en el barrio CD se debe a que:

\footnotetext{
${ }^{5}$ Asimismo, realizamos entrevistas semi-estructuradas a informantes claves; entre ellos funcionarios y trabajadores de gobierno en distintos niveles. Destacamos los encuentros con los directores de los PUI del Oeste y Noreste, la directora de la Agencia Santa Fe Hábitat, la coordinadora del Programa de Regularización Dominial, funcionarios de distintas dependencias vinculados a estos programas, asistentes sociales participes de las relocalizaciones, y referentes del MSLT, entre otros. No obstante, con el fin de mantener el anonimato de los vecinos entrevistados en Coronel Dorrego, hemos conservado solo la inicial de sus nombres, omitiendo sus apellidos. También preservamos el anonimato de algunos funcionarios-trabajadores de dependencias estatales.
} 
-el mismo se encuentra dentro las primeras fases de instrumentalización del PUI del Noreste entre los años 2013-2014. Es decir, que las familias llevan entre tres a cuatro años de residencia en los módulos habitacionales;

-el caso tiene como sustento teórico la entrega de las escrituras del suelo, a partir de la instrumentalización de los Programas de Regularización Dominial. No obstante, esta etapa se encuentra inconclusa en ambos barrios;

-el convenio acuerda la construcción de módulos habitacionales mínimos en forma conjunta con el Movimiento Los Sin Techo de Santa Fe, enmarcando a su vez esta práctica dentro del Programa de Erradicación de Ranchos que dicha organización viene desarrollando;

-en este convenio, la construcción de los módulos habitacionales había de realizarse a partir de modalidades de esfuerzo propio y ayuda mutua por parte los beneficiarios;

-el contexto urbano en donde se enmarca esta relocalización, es acompañado de obras de recualificación y "embellecimiento" urbano, que posiblemente contribuyan a nuevos desplazamientos residenciales de la población empobrecida.

Desde este recorte, indagamos en las condiciones habitacionales preexistentes de las familias al proceso de relocalización; es decir, en la localización inmediata en donde las familias fueron censadas -sobre los reservorios, sobre la traza de la vía, sobre la traza del ferrocarril-.Posteriormente, preguntamos por los desplazamientos previos que atravesaron los integrantes del grupo familiar hasta llegar a esa localización. Sobre este aspecto, preguntamos a su vez por el lugar de nacimiento de los entrevistados, y si son nativos de Santa Fe, o bien de otra localidad (desplazamientos interurbanos). De este modo, buscamos investigar, en los casos en que fue posible, en los desplazamientos de los jefes y jefas de hogar y en los motivos por lo que los mismos decidieron trasladarse hasta la localización previa a la relocalización, así como en los desplazamientos producidos hasta su vivienda actual. Sin embargo, en algunos casos esta cuestión fue definida de manera indirecta en relación con los cónyuges de la jefa y/o el jefe, o los hijos de estos, por imposibilidad de reunirnos directamente con los mismos. Por último, analizamos las condiciones habitacionales posteriores al proceso de relocalización, tanto en el momento inmediato del traslado a las nuevas viviendas como en las variaciones realizadas hasta la fecha en que realizamos las entrevistas. En todos los casos, esta información fue obtenida a partir de las entrevistas personales a las familias y del relevamiento cartográfico de sus viviendas a partir de la información recabada en el trabajo de campo.

Para esto, consideramos las contribuciones sobre la noción de hábitat residencial sustentable (AA.VV, 2004:13-15, 2004르), entendiendo a la misma como un relación que abarca procesos habitacionales complejos, tanto en dimensiones objetivas como subjetivas que incluyen a la vivienda, su entorno inmediato, el conjunto habitacional, el barrio y el contexto urbano mayor. Siguiendo a esta fuente, la habitabilidad es determinada por la relación y la adecuación entre el hombre y el entorno en donde vive, y refiere a cómo cada una de estas escalas es valorada, de acuerdo a un conjunto de factores que se consideran relevantes por el habitante (AA.VV, 2004푸; Núñez, 2013). En este marco, la apropiación del espacio sólo puede lograrse en la interrelación de esas cualidades, y desagregarse a los efectos analíticos en habitabilidad espacial (dimensionamiento, distribución y uso), habitabilidad psicosocial (asociada a las características socio-económicas y culturales, como la identidad de lugar), habitabilidad térmica (temperatura y humedad en la vivienda), habitabilidad acústica, habitabilidad lumínica (iluminación natural), seguridad y mantención (durabilidad y capacidad de administración según las características del medio geográfico, seguridad estructural, etc.).

A partir de estos aportes, seleccionamos las variables e indicadores ${ }^{6}$ habitacionales para llevar adelante la investigación, los que clasificamos en: 1) Tipo de familia; 2) Vivienda; 3) Saneamiento ambiental; 4) Organización social; 5) Percepciones; y 6) Desplazamientos. No obstante, debido a las limitaciones de espacio de este trabajo, expondremos a continuación algunos de los indicadores de vivienda. En este marco, hemos caracterizado estos últimos en tres grupos: a) Condición Habitacional: promedio de personas por vivienda, cantidad de habitaciones de la vivienda, disponibilidad de cocina y baño; características de la vivienda y estado de conservación, calidad constructiva de la vivienda, materiales, pisos y techos, habitabilidad y materialidad y condiciones de hacinamiento personal; b) Condiciones de Tenencia: tipo de documento de dominio de suelo, (escritura de dominio, boleto de compraventa, certificado de cancelación de deuda, constancia de ocupación, acta de tenencia, acta de adjudicación, acta de pre-adjudicación, entre otros); y c) Créditos y Ampliación: motivo y tipo de modificación en la vivienda ;tipo de financiamiento para la ampliación (préstamos, hipotecas, microcréditos para materiales, microcréditos para mano de obra, y

\footnotetext{
${ }^{6}$ Siguiendo a Wautiez y Reyes (2002:19-51), "un indicador es una herramienta para medir y comunicar información, que permite representar un conjunto de datos y visualizar los cambios generados en el tiempo, si se ha mejorado o no."
} 
distintas modalidades de financiamiento para la ampliación, refacción y otras modificaciones, y accesibilidad a otros servicios: telefonía, cable, entre otros.

\section{DE LA CHAQUEÑADA A CORONEL DORREGO}

Al noreste de la ciudad y en proximidad a la zona de costera, se constituyó el Barrio Chaqueño o la Chaqueñada -en adelante $\mathrm{CH}$. Históricamente, esta zona se caracterizó en sus inicios por ser una zona de quintas próximas a estaciones intermedias del ferrocarril para la carga y descarga de pasajeros. Algunos vecinos del barrio, según nuestro trabajo de campo, remiten a la ocupación inicial de estos suelos por familias empobrecidas que provenían de norte del país en busca de trabajo, justamente en tren y principalmente de Chaco, y construían sus primeras viviendas próximas a estas estaciones. Las familias que fueron censadas para la relocalización residían en situaciones de precariedad, localizadas sobre la traza vacante de suelo al margen de la vía del ferrocarril y sobre la traza prevista para la calle Güemes, en varios casos por más de dos décadas. Sin embargo, estos terrenos permanecían vacantes estando destinado a traza de la circunvalación, que no llego a concretarse (Mapa №1).

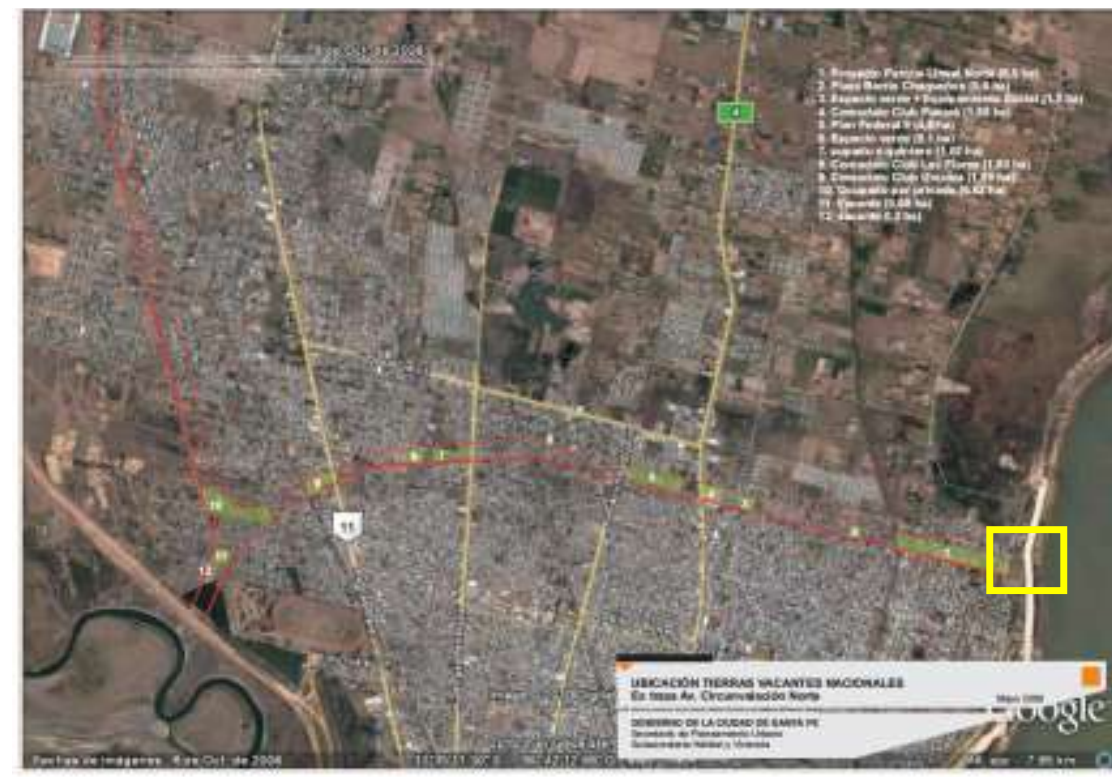

Mapa $N^{\circ}$ 1: Suelo vacante del ex proyecto de circunvalación, Santa Fe.

Fuente: Gobierno de la Ciudad de Santa Fe, 2009.

Previa a la relocalización en CD, las familias no disponían de los servicios públicos de agua potable, electricidad, gas y cloaca; y las calles tampoco se encontraban pavimentas. Sin embargo, las familias resolvían esta situación a partir de conexiones domiciliarias precarias para obtener estos servicios. Según nuestro trabajo de campo:

- la mayoría de los hogares censados para la relocalización eran hogares multipersonales no conyugales -59,4\%- con jefa de hogar mujer entre 25 a 65 años -82,4\%-,

- Un predominio de viviendas de tipo material $-57,8 \%-$,

- Si bien más de un $40 \%$ de las familias que residían sobre la Calle Guemes tenían más de 5 miembros, sobre la vía del ferrocarril aproximadamente un 70\% disponía entre 3 a 4 personas por vivienda,

el 30\% de las viviendas localizadas sobre calle Güemes disponía de una habitación, un $96,1 \%$ tenía baño, un $70 \%$ cocina, y un $20 \%$ agua y electricidad mediante servicio público. No obstante, un $65 \%$ de las viviendas restantes, tenían entre 2 a 4 habitaciones, y solo un porcentaje cercano al $20 \%$ tenía agua y electricidad,

las familias que residían sobre la vía en su mayoría también disponía solo de una habitación $-60 \%-$, y un $40 \%$ tenían entre 3 a 4 habitaciones. A pesar de estas condiciones, las 11 viviendas disponían de agua y electricidad, según el relevamiento,

- $\quad$ el $24 \%$ de los hogares censados en la segunda etapa de relocalización, tenían boleto de compraventa.

En este contexto, en el año 2012, y enmarcado en la Secretaría de Hábitat de la Provincia, tiene lugar la firma del Convenio Marco de "Colaboración y articulación de políticas de hábitat y vivienda"-en adelante 
Convenio Marco gobierno/MLST- El mismo fue materializado entre tres instituciones: la DPYyU por parte de organismos provinciales, a través de la Dirección de Emergencia Habitacional; la Agencia Hábitat Santa Fe por parte de aparatos municipales; y el MLST, como organización social. ¿En qué consistió este convenio? Sucintamente, en la articulación conjunta entre estas instituciones para llevar adelante la relocalización de un grupo de familias asentadas desde hace décadas en algunos barrios empobrecidos de la ciudad, en los que se incluye CD dentro del PUI del Noreste. Esto fue posibilitado mediante la siguiente articulación de tareas:

- La DPYyU, a través de Dirección de emergencia habitacional realizó los aportes financieros para la construcción de los módulos habitacionales; así como también llevó a cabo la inspección de las obras. Es decir, cumplió la función financiera y de control de obra.

-La Agencia Santa Fe Hábitat, en el marco del programa de Regularización Dominial, estaría encargada de cumplir la parte normativa y jurídica de este proceso, a partir de la regularización de los lotes.

- EI MLST, aportaría la mano de obra especializada para la ejecución de los módulos habitacionales; designando a las familias beneficiarias, y realizando un seguimiento en este proceso. A su vez, estuvo encargada de mediar el trabajo con las familias para posibilitar la relocalización. Sin embargo, esta organización tercializó la mano de obra y esta fue llevada a cabo por la Empresa de Premoldeados Bertone.

\section{$4 \quad$ LAS VIVIENDAS MINIMAS}

Los terrenos de la relocalización fueron de aproximadamente $170 \mathrm{~m} 2$ y las viviendas abrían de ser asignadas tanto en base a la cantidad de integrantes del grupo familiar, como en cuanto a las características de la vivienda afectada. Las soluciones habitacionales varían, en este sentido, entre el prototipo A1, proyectado para que convivan hasta 3 integrantes en un ambiente, que comprende la cocina, el estar, comedor y dormitorio, y un baño; y el prototipo A2, de dos ambientes para familias de 4 a más integrantes, donde lo que varía es la disponibilidad de un dormitorio aparte, además del baño y el otro ambiente. En ambos prototipos queda prevista una platea en la parte de atrás para la ampliación a otro dormitorio. A su vez, ambos disponen de bajo-mesada en el sector de cocina (Cuadro $\mathrm{N}^{\circ} 1$ )

\begin{tabular}{|c|c|c|c|c|c|c|c|c|c|c|c|c|c|c|}
\hline & & & & & Proto & ipo & vien & & & & Sup & rficie & & \\
\hline & 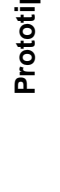 & 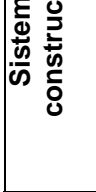 & 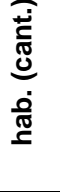 & 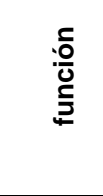 & 丞 & 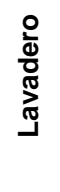 & פ̊ำ & $\frac{\frac{\pi}{\frac{\pi}{2}}}{\frac{\pi}{\sigma}}$ & 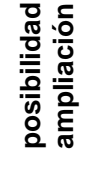 & 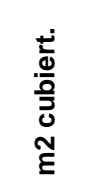 & 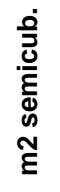 & $\begin{array}{l}\bar{\varpi} \\
\stackrel{\Xi}{0} \\
\text { กิ }\end{array}$ & 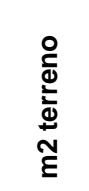 & $\begin{array}{l}\text { ᄒे } \\
\text { o } \\
\text { ơ } \\
0 \\
0\end{array}$ \\
\hline 岂 & $\bar{\varangle}$ & 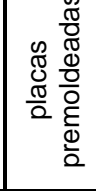 & 1 & $\begin{array}{l}\text { Estar- } \\
\text { comd- } \\
\text { coc.- } \\
\text { dorm. }\end{array}$ & si & si & si & no & platea & $28 \mathrm{~m} 2$ & - & $28 \mathrm{~m} 2$ & $\begin{array}{c}169 m \\
2\end{array}$ & $\begin{array}{l}\text { Año } 2013 \\
\$ 190.060\end{array}$ \\
\hline $\bar{\Omega}$ & $\tilde{x}$ & 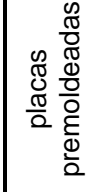 & 2 & $\begin{array}{l}1 . \\
\text { Estar- } \\
\text { comd- } \\
\text { coc. } \\
\text { /dorm. }\end{array}$ & si & si & si & no & platea & $42 \mathrm{~m} 2$ & - & $42 \mathrm{~m} 2$ & $\begin{array}{c}169 m \\
2\end{array}$ & $\begin{array}{c}\text { Actualización } \\
\text { Año } 2014 \\
\text { \$330.944 }\end{array}$ \\
\hline
\end{tabular}

Cuadro $\mathrm{N}^{\circ}$ 1: Convenio Marco gobierno/MLST: prototipo universal de vivienda, Al y A2, funcionalidad. Santa Fe, 2012-2014

Fuente: elaboración propia

En este marco, la Secretaría de Estado del Hábitat de la Provincia de Santa Fe expresa que el prototipo universal de vivienda fue diseñado considerando:

“...una nueva alternativa al modo de pensar la vivienda social, que contemple criterios de universalidad desde su concepción, flexibilidad de crecimiento, diversidad en su materialización (tipológica y formal) y eficiencia energética (...) La idea fuerza que acompaña esta iniciativa es que las familias beneficiarias de planes oficiales no son todas iguales. Por el contrario todas presentan sus características particulares, no solo en el momento de recibir la vivienda sino también a futuro. En este sentido, la principal virtud del nuevo prototipo es la capacidad de poder adaptarse a cada situación particular (...) A partir de un núcleo básico de $\mathbf{3 0}$ m2 (baño, cocina, 
lavadero y espacio común de $16 \mathrm{m2}$ ), se cuenta con diversas alternativas de crecimiento, según la ubicación en el terreno y la necesidad particular."7

Una primera contradicción surge de esta premisa: si bien es cierto que familia tiene sus particularidades, es imposible pensar que la solución al problema de la vivienda (Pradilla, 1984) puede resolverse en un núcleo básico de 30m2, más allá de las posibilidades de crecimiento, modificación y ampliación que las familias puedan realizar. Tampoco es factible suponer esta afirmación solo a las familias beneficiarias de planes sociales, dado que estas situaciones no son recomendables para ninguna familia, a pesar de la condición social que se encuentre. En consonancia, desde el vamos se proyecta que convivan en una misma habitación varios integrantes del grupo familiar, o todos, dependiendo el caso; así como que también coexistan diversas actividades, incluyendo las de descanso, alimentación, reproducción sexual, recreación, entre otras. Esto implica que los hijos duerman en el mismo local que sus padres, u otras situaciones complejas según la composición del grupo familiar. De modo similar, el prototipo de un dormitorio implica que parte de la familia reproduzca sus condiciones de vida en la misma habitación multifunción con todas las actividades antes mencionadas, realizadas en forma simultánea por los diversos integrantes del grupo familiar. Bajo estas premisas, en el Cuadro $\mathrm{N}^{\circ} 2$ observamos la vinculación de las cuatro cualidades propuestas a nivel discursivo y su relación con los dos prototipos.

\begin{tabular}{|c|c|c|c|c|}
\hline \multirow{3}{*}{ 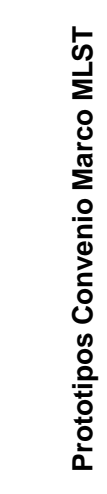 } & \multicolumn{4}{|c|}{ Prototipo Universal de Vivienda } \\
\hline & Universalidad & $\begin{array}{l}\text { Flexibilidad de } \\
\text { crecimiento }\end{array}$ & $\begin{array}{l}\text { Diversidad formal y } \\
\text { tipológica }\end{array}$ & $\begin{array}{l}\text { Eficiencia } \\
\text { energética* }\end{array}$ \\
\hline & $\begin{array}{l}\text { Espacios funcionales } \\
\text { capaces de contener la } \\
\text { pluralidad de } \\
\text { condiciones familiares } \\
\text { (capacidades diferentes, } \\
\text { ancianidad, niñez, } \\
\text { embarazo, etc.) }\end{array}$ & $\begin{array}{l}\text { Multiplicidad de variables } \\
\text { de crecimiento que } \\
\text { atienden a las demandas } \\
\text { del grupo familiar, y las } \\
\text { complementarias a la } \\
\text { vivienda (local comercial, } \\
\text { taller, estudio, etc.) }\end{array}$ & $\begin{array}{l}\text { Captación de recursos } \\
\text { naturales, económicos y } \\
\text { humanos disponibles en el } \\
\text { territorio, oportunidad para } \\
\text { el crecimiento de } \\
\text { economías locales } \\
\text { (cooperativas, micro } \\
\text { emprendimientos y } \\
\text { empresas) }\end{array}$ & $\begin{array}{c}\text { Políticas orientadas a } \\
\text { la generación de un } \\
\text { hábitat sostenible, } \\
\text { aprovechamiento y } \\
\text { utilización de } \\
\text { energías renovables. }\end{array}$ \\
\hline Al & $\begin{array}{l}\text { no contempla (excepto } \\
\text { en casos particulares) }\end{array}$ & $\begin{array}{c}\text { expansión a } 1 \text { dormitorio } \\
\text { sobre platea y galería o } \\
\text { cochera sobre lateral }\end{array}$ & $\begin{array}{l}\text { construcción a cargo de } \\
\text { empresa de premoldeados }\end{array}$ & no contempla \\
\hline A2 & $\begin{array}{l}\text { no contempla (excepto } \\
\text { en casos particulares) }\end{array}$ & $\begin{array}{l}\text { expansión a galería o } \\
\text { cochera sobre lateral }\end{array}$ & $\begin{array}{l}\text { construcción a cargo de } \\
\text { empresa de premoldeados }\end{array}$ & no contempla \\
\hline
\end{tabular}

Cuadro $\mathrm{N}^{\circ}$ 2: Relación entre los argumentos del prototipo universal de vivienda y su materialización en los PUl. Fuente: elaboración propia.

Tal como vemos, ninguno de los aspectos conceptualizados es contemplado en su totalidad: exceptuando algunos casos particulares, tipológicamente los prototipos universales no incorporan ninguna novedad respecto a la tipología tradicional de vivienda social; tampoco lo hacen en relación al uso de energías sustentables, exceptuando en la construcción de las nuevas viviendas en el barrio las Lomitas que incorporan el uso de calefones solares. A su vez, si bien es cierto que el prototipo Al contempla la posibilidad de ampliación sobre la platea, esta se encuentra proyectada desde el vamos, y no sobre los fundamentos de la flexibilidad de crecimiento de acuerdo a los requisitos de cada familia. Por ejemplo, no hay mayores posibilidades de expansión de las nuevas habitaciones más que hacia atrás o bien sobre uno de los laterales. Respecto a la posibilidad de construcción sobre la planta alta, esta se ve limitada en ambos prototipos dado que la cubierta es de chapa. Por último, los principios de ayuda mutua y esfuerzo compartido convenidos, se ven quebrantados al estar las viviendas construidas por una empresa de premoldeados, donde las placas son montadas por obreros especializados de la propia empresa:

\footnotetext{
${ }^{7}$ En https://www.santafe.gov.ar/index.php/web/content/view/full/173945 (Última visita 8/11/17)
} 
Este último acápite se basa en algunas de las reflexiones realizadas en base a las 23 entrevistas personales -de un total de 37 familias relocalizadas- que realizamos en CD, cuyas viviendas fueron afectadas totalmente para la operación de apertura de calles y esponjamiento. En este sentido, el proceso de relocalización fue programado en tres etapas y realizado entre los años 2013 y 2014: 1) una primer etapa en donde se relocalizaron 11 familias para la apertura de calle Güemes; 2) una segunda etapa de 16 familias para la apertura de la colectora Dorrego; y $\mathbf{3}$ ) una tercer y última etapa de $\mathbf{1 0}$ familias para el ensanche de pasillos y consolidación de plazoletas barriales internas (Mapas $\mathrm{N}^{\circ} 2$ y 3). El nuevo loteo comprendió 38 parcelas, y una de estas se dejó prevista para la construcción de la Vecinal Coronel Dorrego.

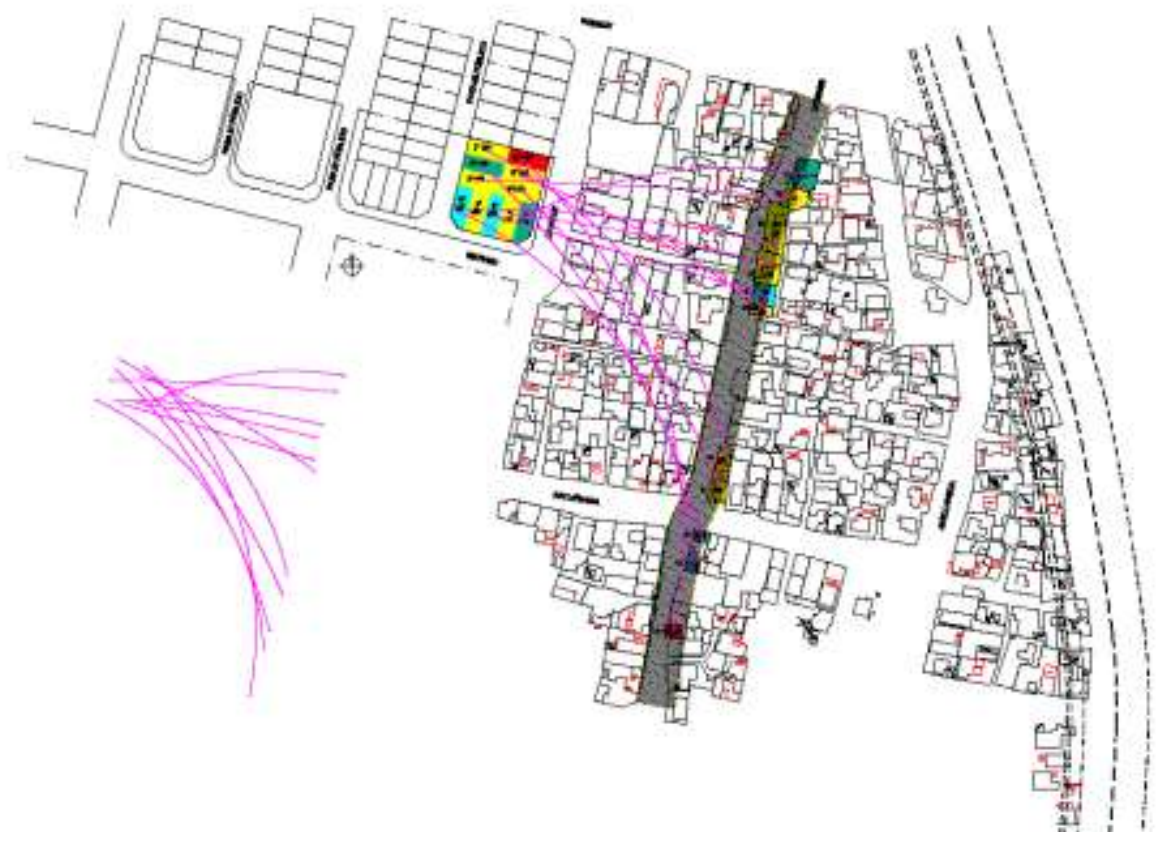

Mapa № 2: Relocalizaciones de población: $1^{\circ}$ etapa. CD, Santa Fe, 2013.

Fuente: elaboración propia en base a información recabada.

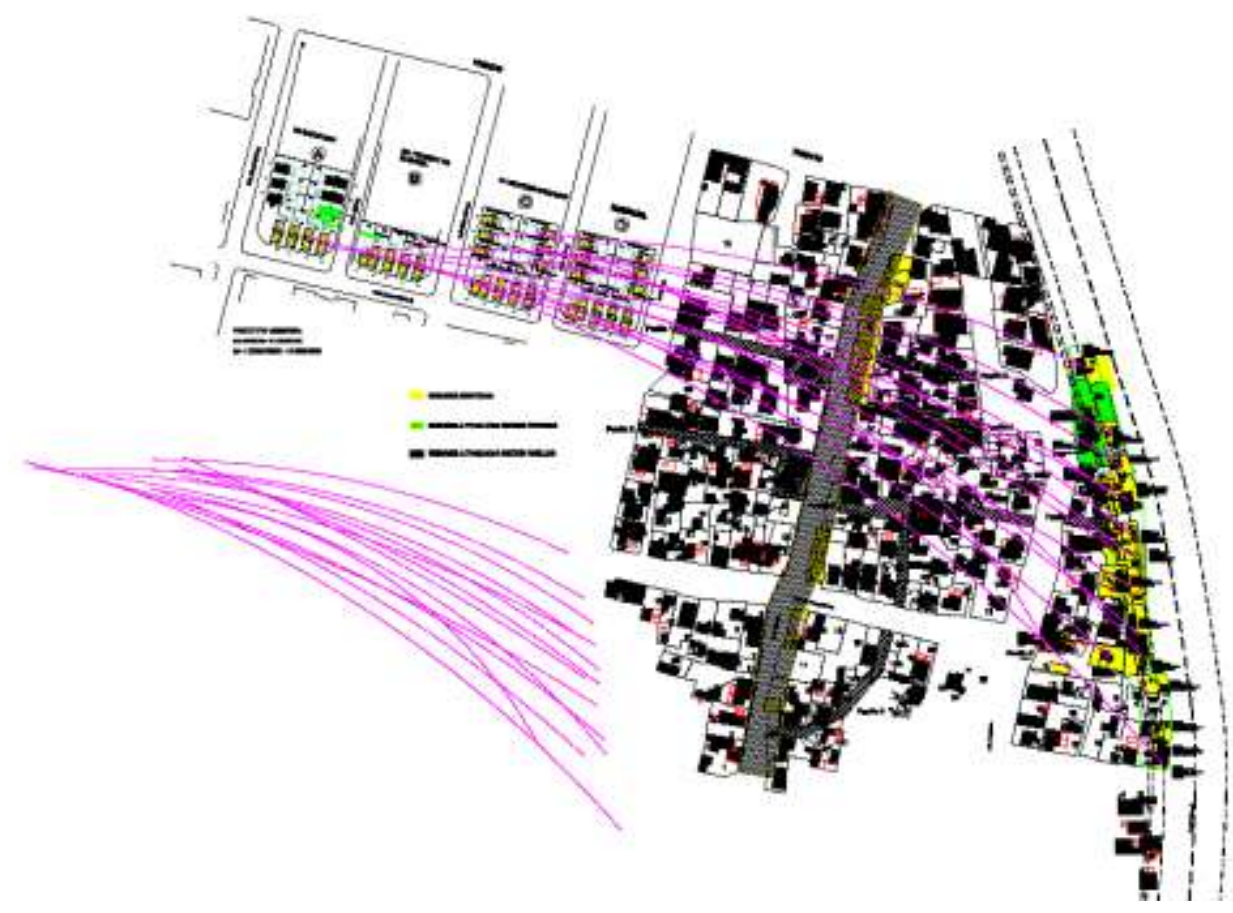

Mapa N 3: Relocalizaciones de población: $2^{\circ}$ etapa. CD, Santa Fe, 2013.

Fuente: elaboración propia en base a información recabada. 
Hemos comprendido este proceso en tres etapas: i)el relevamiento social previo a la relocalización; ii) la mudanza de las familias y sus pertenencias realizadas por el gobierno en camiones, en algunos casos con un aviso previo de 3 meses, y en otros casos, el día anterior, y en paralelo, iii) la demolición de la viviendas que, según relatan nuestros entrevistados, fue realizada frente a los propios vecinos. Es decir, que las mismas se encontraban presentes cuando demolían sus casas ${ }^{8}$. No obstante, las mudanzas fueron realizadas en meses distintos de manera individual. En este sentido, la mayoría de los entrevistados coinciden en considerar que: "[los] censaron-[los] sacaron-desarmaron todo-[los] cruzaron":

\begin{abstract}
"Nos iban sacando, por ejemplo terminaban las casitas, () y ya te iban y te decían, 'para mañana tenes que tener todo preparado' () ejemplo llegaban a la tarde, y al otro día a las 7 de la mañana ya llegaba el camión y ya tenías que cargar todo y venir. () a mí me tuvieron como 3 meses con todo preparado para venirme". (Entrevista personal a B., jefa de hogar)

"Tenía dos cimientos, uno ya estaba terminado, y otro aparte atrás que se estaba a medio terminar. Los materiales algo nomas, no mucho. Nos dijeron que nos iban a dar dos días para que saquemos algunas cosas y ese mismo día que nos fueron a buscar la maquina se encargó de tirar todo en ese mismo momento mientras estábamos sacando las cosas". (Entrevista personal a Ev., jefa de hogar)
\end{abstract}

En este marco, del total de familias entrevistadas, 10 de ellas han recibido una vivienda prototipo A1, y 13 familias una vivienda A2. Estas fueron otorgadas de acuerdo a la cantidad de integrantes del grupo familias y las condiciones constructivas de las viviendas anteriores. En el Mapa $N^{\circ} 4$ observamos esta distribución.

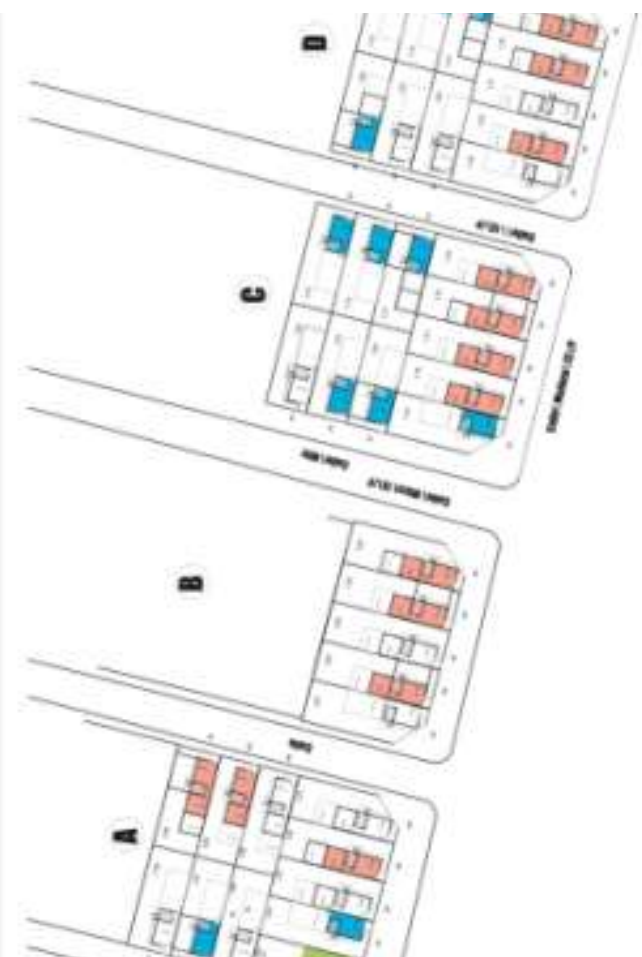

Mapa N 3: Prototipos A1 y A2: localización según tipo, CD, Santa Fe, 2013/2014.

Fuente: elaboración propia en base a información recabada.

Tal como vemos en el Cuadro $\mathrm{N}^{\circ} 2$, en la residencia anterior en barrio $\mathrm{CH}$, la mayoría de las viviendas era de tipo material -el 64,7\%-, y un porcentaje importante -42,1\%- disponía de 3 habitaciones. Esto implicó, que estas familias hayan autoconstruido con mucho esfuerzo sus propias viviendas, a partir de la prolongación de su jornada laboral y utilizando materiales de desecho (Pradilla, 1984). A su vez, un 10,6\% tenían 4 o más habitaciones, y un $26,3 \%$ dos habitaciones, siendo solo un $21 \%$ las de tipo monoambiente como el prototipo A1 en CD. Es decir, que en general se trataban de viviendas compartimentadas en cuanto a la distribución de los espacios. No obstante, el $94,7 \%$ de las familias disponía de cocina y baño propio dentro de su vivienda. En este marco, más de la mitad de las familias en $\mathrm{CH}$ tenían boleto o algún tipo de documentación de su vivienda en el momento en que la adquirieron.

\footnotetext{
${ }^{8}$ Algunas familias remiten a que esta situación fue realizada en simultáneo para evitar que nuevas familias se apropiaran de estas viviendas. Esto fue corroborado a partir de lo conversado con una de nuestras informantes claves: "...iban juntos el camión de mudanza y el camión que iba a destruir las casa donde vivían, ¿para qué? Para que no la usurpen, sino era un trabajo en vano" (Asistente social de la relocalización en Coronel Dorrego A1-01 21/02/2017)
} 


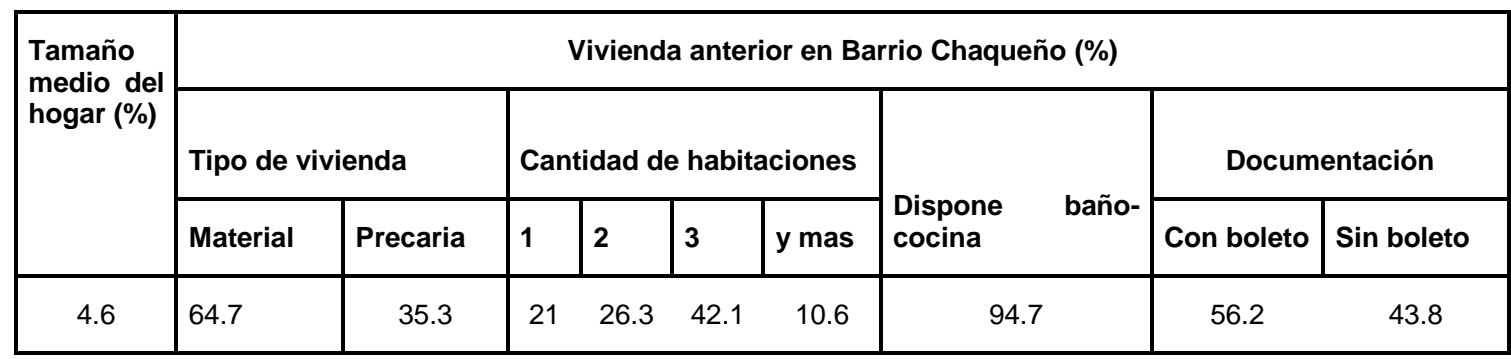

Cuadro $\mathrm{N}^{\circ}$ 2: Viviendas: tipo de vivienda y condiciones habitacionales previas (\%) en barrio Chaqueño (CH), Santa $\mathrm{Fe}$.

Fuente: elaboración propia en base entrevistas realizadas.

En la mayoría de los casos, los entrevistados aluden a la voluntad realizada por el grupo familiar en la construcción y ampliación de su vivienda en $\mathrm{CH}$, o bien en el esfuerzo económico invertido para poder solventar estos gastos. En este marco, varios de ellos describen a las viviendas en $\mathrm{CH}$ como "más grandes", y mencionan que antes estaban "más cómodos" en cuanto a la superficie que estas tenían:

\begin{abstract}
"Ya de 26 años te duele, ¿viste? Aparte todo nuevo, habíamos empezado a comprar los pisos, hacer todo. Pero yo hacía para la comodidad (...) de vivir bien, porque ya estábamos cansados de vivir mal siempre. $Y$ con una economía que trabajamos bien, es decir, ¿y si no nos sacan? y bueno llego el momento que nos sacaron" (Entrevista personal a B., jefa de hogar)
\end{abstract}

"Nos dijeron que íbamos a tener la comodidad o se iba a parecer más o menos a lo que teníamos. Cuando llegamos acá nos encontrábamos con que no tenía... está bien, es cómoda la casa, pero ella [refiere e tía E.] en su casa tenía dos habitaciones, y acá no tiene. Allá en mi casa también, tenía casi todo a medio terminar y ahora tengo que empezar de nuevo" (Entrevista personal a Ev., jefa de hogar)

"Yo tenía más cosas (...) pero las deje allá porque acá no las podía traer porque no entraban" (Entrevista personal a E., jefa de hogar)

Del total de familias entrevistadas, el $52,2 \%$ sí amplió sus viviendas (Mapa $N^{\circ} 4$ ), realizando, en su mayoría, una habitación más. Aquellas familias que han ampliado a 3 habitaciones, la tercera corresponde a un garaje. Del total de familias que pudo ampliar, un $63,6 \%$ lo hizo con fondos propios, y un $36,4 \%$ lo hizo a través del préstamo ARGENTA ${ }^{9}$ otorgado a las madres, principalmente para aquellas en CD que perciben la asignación universal por hijo, la pensión de los 7 o más hijos o bien por la jubilación (Cuadro $N^{\circ} 3$ ). En todos los casos en donde se ha ampliado la vivienda (Mapa $N^{\circ} 5$ ) a partir de préstamos han sido las mujeres jefas de hogar quienes los han solicitado:

"Hace dos año me salió la jubilación y me hice esa pieza con mi trabajo, y aquella del fondo con los préstamos de la Argenta" (Entrevista personal a M., jefa de hogar jubilada)

"Saque un préstamo en el banco, no la termine, hice un toque nomás. Después para techar, un día de tormenta se le voló al vecino todas las chapas de ahí enfrente, $y$ todas las chapas pasaron para este lado (...) y bueno, las chapas esas agarre para techar la pieza que tengo. Y estamos con esas nomas. Y ahora estoy esperando a ver si la asistente me ayuda para hacer la otra en el fondo. Porque somos seis nosotros" (Entrevista personal a N., jefa de hogar)

\begin{tabular}{|c|c|c|c|c|c|c|c|c|c|c|}
\hline \multirow{3}{*}{$\begin{array}{c}\text { Tamaño } \\
\text { medio } \\
\text { del } \\
\text { hogar } \\
(\%)\end{array}$} & \multicolumn{10}{|c|}{ Vivienda actual Coronel Dorrego } \\
\hline & \multicolumn{2}{|c|}{$\begin{array}{c}\text { Prototipo } \\
\text { (cant.) }\end{array}$} & \multirow{2}{*}{$\begin{array}{c}\text { No } \\
\text { amplió } \\
(\%)\end{array}$} & \multicolumn{4}{|c|}{ Si amplió (\%) } & \multicolumn{3}{|c|}{ Fondos para la ampliación (\%) } \\
\hline & A1 & A2 & & 1D & $2 \mathrm{H}$ & $3 \mathbf{H}$ & $4 \mathrm{H}$ & $\begin{array}{l}\text { Fondos } \\
\text { propios }\end{array}$ & Préstamo & $\begin{array}{c}\text { Tipo de } \\
\text { préstamo }\end{array}$ \\
\hline 4.9 & 10 & 13 & 47.8 & 41.6 & 25.1 & 33.3 & - & 63.6 & 36.4 & Argenta \\
\hline
\end{tabular}

${ }^{9}$ Argenta es un programa de créditos personales que tiene por destinatarios a Jubilados y Pensionados del Sistema Integrado Previsional Argentino/SIPA, titulares de Asignación Universal Por Hijo/AUH (Madres o padres viudos), del Sistema Único de Asignaciones Familiares/SUAF (Rangos 1 y 2), de Pensiones No Contributivas/PNC (Madres de siete o más hijos, invalidez y Adulto Mayor), y de Pensión Universal para el Adulto Mayor (PUAM). Para mayor información se recomienda consultar la pag. Web del ANSES http://argenta.anses.gob.ar/seccion/objetivos-del-programa-30. 
Cuadro $\mathrm{N}^{\circ}$ 3: Viviendas: prototipos (cantidad), ampliación y fondos económicos para la ampliación (\%) en CD, Santa Fe,2017. Fuente: elaboración propia en base entrevistas realizadas.
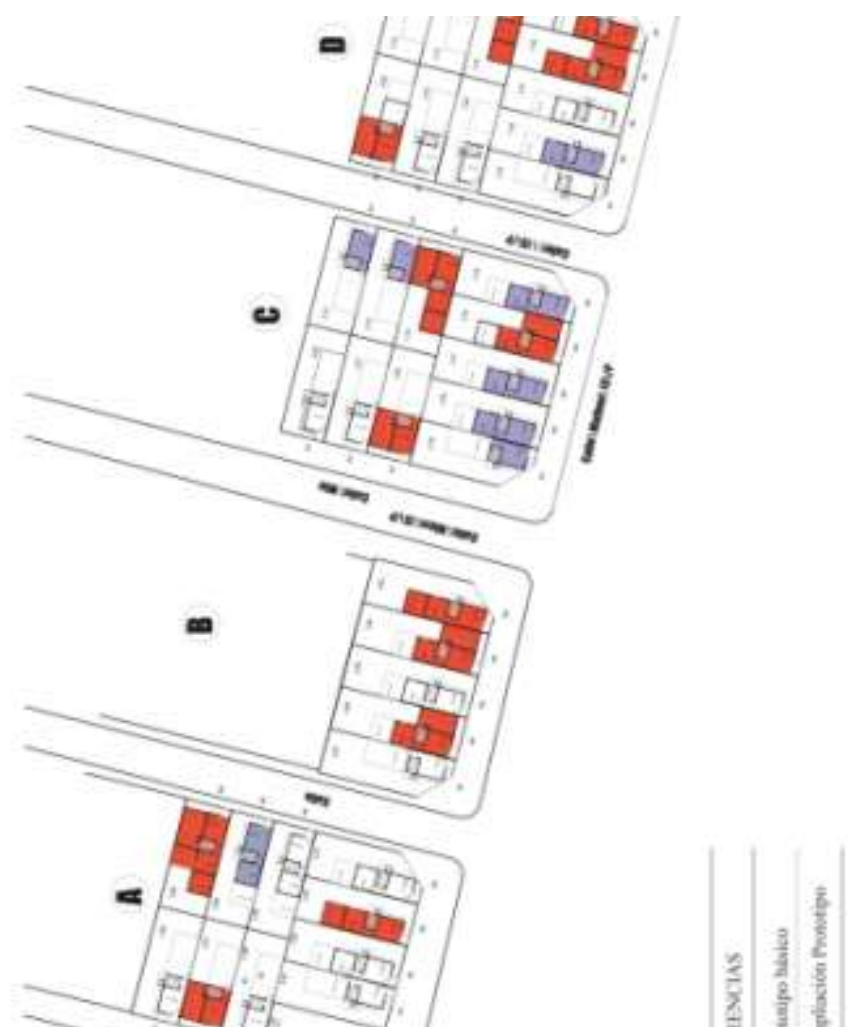

Mapa $\mathrm{N}^{\circ}$ 4: Familias que ampliaron sus viviendas y aquellas que las conservaron, $\mathrm{CD}$, Santa $\mathrm{Fe}$.

Fuente: elaboración propia en base a información recabada.

En este contexto, es preciso señalar, que si bien esta política no reprodujo hacinamiento del hogar al momento de la entrega de las viviendas, dado que a pesar de la conformación familiar en la anterior vivienda, cada hogar recibió un núcleo básico. Sin embargo, si hemos detectado que en CD esta situación se ha revertido en algunos casos, con la llegada de nuevos integrantes al grupo familiar. No obstante, no podemos dejar de vincular esta situación con el hacinamiento personal que estas condiciones habitacionales presentan para las familias, y que al momento de la pre-adjudicación de las viviendas, sí ha sido reproducida por el propio gobierno, tal como veremos a continuación.

Si retomamos la definición del INDEC de hacinamiento personal, esta condisera aquellos hogares en donde residen más de 3 personas por cuarto, exeptuando con ello a la habitación destinada para cocina y baño. $Y$ tal como vimos en el diseño del prototipo $\mathrm{A} 1$ en principio, desde el vamos se se proyecta que convivan en una misma habitación 3 integrantes del grupo familiar ${ }^{10}$. Este prototipo solo contempla dos habitaciones: un baño aparte y una habitación de multiples usos para que coexistan diversas actividades, incluyendo las de descanso, alimentación, reproducción sexual, recreación, entre otras. Esto implica que los hijos duerman en el mismo local que sus padres, u otras situaciones complejas según la composición del grupo familiar. En palabras de nuestras entrevistadas:

"Acá [refiere a la habitación multifunción] ocupábamos para dormitorio, y cocina un pedacito" (Entrevista personal a M., jefa de hogar jubilada)

"Nos habian dicho que por ella [la hermana tiene dificultades motrices] tenía que tener una pieza sola. Pero nunca vinieron a solucionar ese problema. Nosotros ahora estamos todos juntos." (Entrevista personal a M., hija de jefe de hogar que nació en Barrio Chaqueño)

De modo similar, el prototipo de un dormitorio implica que parte de la familia reproduzca sus condiciones de vida en la misma habitación multifunción con todas las actividades antes mencionadas (Mapa №6),

${ }^{10} \mathrm{Si}$ retomamos la propuesta de la propia política urbana establecida desde aparatos estatales antes vista, recordemos que el prototipo A1 está proyectado para que convivan hasta 3 integrantes en un ambiente, que comprende la cocina, el estar, comedor y dormitorio, y un baño; siendo el prototipo A2 de dos ambientes y considerado para familias de 4 a más integrantes, donde lo que varía es la disponibilidad de un dormitorio aparte, además del baño y el otro ambiente. 
realizadas en forma simultánea por los diversos integrantes del grupo familiar: "...porque en realidad tres familias para un solo terreno es mucho. Más que nada cuando uno tiene chicos, no tenes tu espacio" (Entrevista personal a G., cónyuge de jefe de hogar). Resumiendo, en el momento de la relocalización a las nuevas viviendas, prácticamente todos los hogares, exceptuando los unipersonales, presentan hacinamiento del hogar. En consonancia, en relación a la situación actual en CD, y contemplando los casos en donde las familias pudieron ampliar sus viviendas, la mayoría de los hogares presenta hacinamiento de dos tipos. Por un lado, en aquellos hogares que aún no pudieron ampliar sus viviendas y que desde el vamos ya tenían hacinamiento en las nuevas viviendas. Por otro lado, se trata de aquellos hogares a lo que se les han incorporado nuevos integrantes del núcleo familiar, ya sea por nacimiento de un nuevo integrantes o bien por la conformación de la familiar extendida: " $Y$ ahora que no tienen donde vivir los otros [su hermana, el marido y el hijo de estos], se lo ofrecí." (Entrevista personal a Ev., jefa de hogar).En este marco y en base al diseño de los prototipos que contemplan una platea para su futura ampliación, queda presumido que las propias familias amplíen sus viviendas mediante la autoconstrucción. Sin embargo, recordemos que se trata de población empobrecida, en su mayoría sin empleo, cuestión que hace dificultoso en muchos de las casas esta posibilidad:

\begin{abstract}
"Esta tiene un dormitorio, nosotros la tenemos que ampliar, de a poquito (...) porque no está fácil la mano" (Entrevista personal a L. S., jefa de hogar)

"Y de a poco empezamos a tratar de levantar una pieza más porque como somos muchos (...) pero quedamos ahí con la construcción. Quedo él [refiere al marido] sin trabajo. Nos manejamos ahora haciendo cosas de panadería, el sale a vender a la calle, y apenas sacamos justo para comer en el día, y los pañales y leche." (Entrevista personal a M., cónyuge de jefe de hogar)
\end{abstract}

Por último, en cuanto a las condiciones de entrega de las viviendas, varios vecinos mencionan los inconvenientes constructivos en el momento de la recepción, o bien el incumplimiento por parte de aparatos estatales en regresar a solucionar algunos problemas constructivos o bien a completar la falta de algunos materiales de la vivienda (vidrios, grifos, aberturas):

"Mi mama si tuvo que hacer muchas modificaciones acá porque cuando le entregaron la casa tuvo muchos problemas con la cámara, las cosas que perdian agua" (Entrevista personal a V., hija de jefa de hogar)

"No vinieron más, ( ) nos prometieron el cielorraso y la cerámica, que bueno, eso estaba en el contrato. () Pero no aparecieron más asique..." (Entrevista personal a G., cónyuge de jefe de hogar)
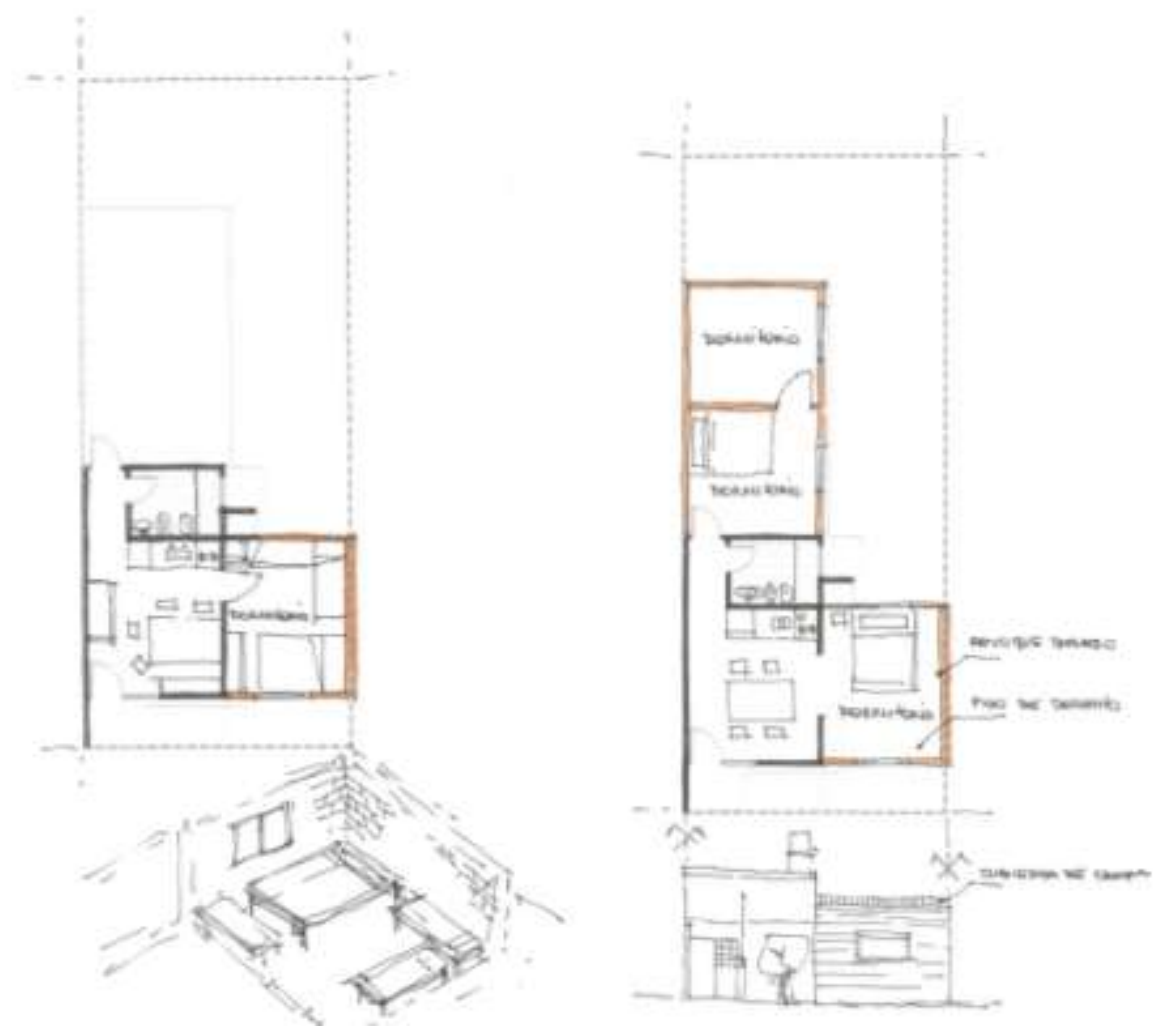

Mapa $N^{\circ}$ 5: Familias que ampliaron sus viviendas a través de préstamos otorgados a las jefas de hogar, CD, Santa Fe, 2017. Fuente: elaboración propia en base a información recabada. 


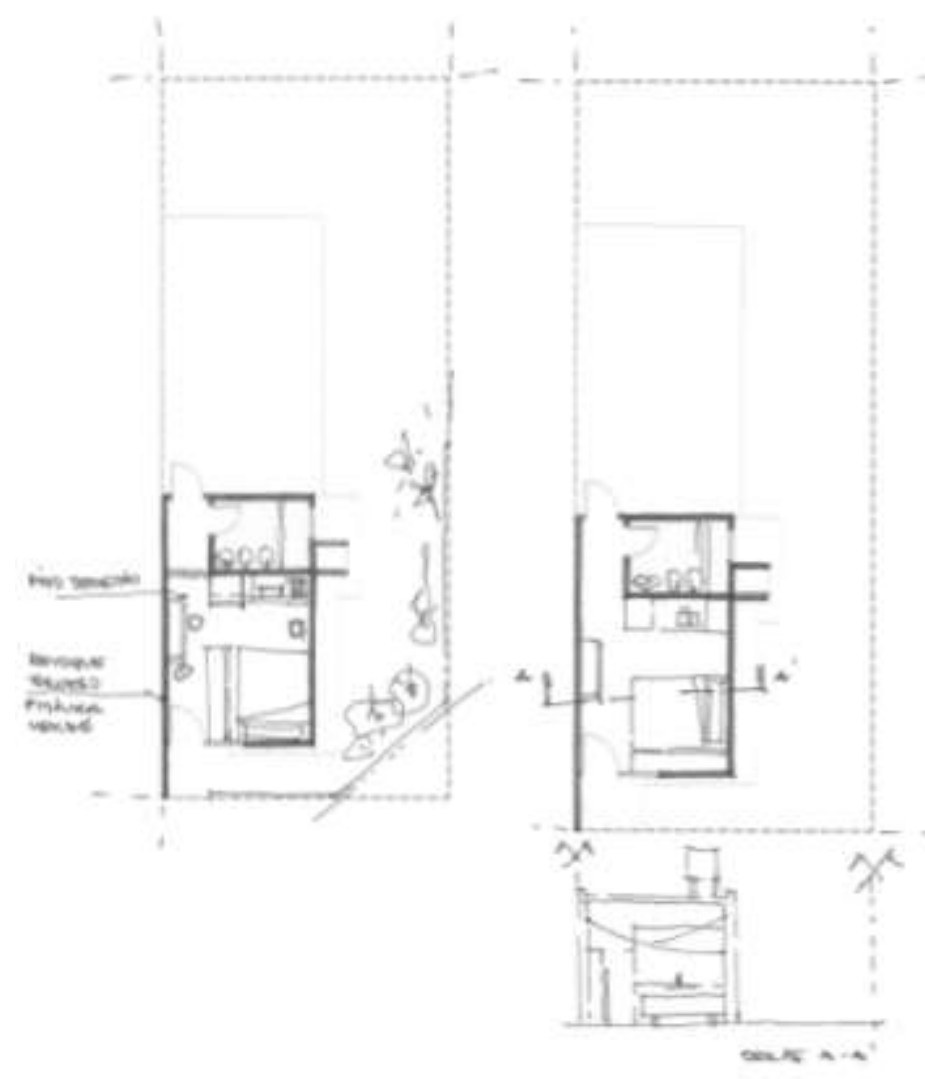

Mapa $\mathrm{N}^{\circ}$ 6: Familias con hacinamiento personal que no ampliaron sus viviendas, CD, Santa Fe, 2017. Fuente: elaboración propia en base a información recabada.

\section{REFLEXIONES FINALES}

En primer lugar, y de acuerdo al contexto latinoamericano, década tras décadas podemos corroborar como es reproducido el corpus hegemónico de los Ol sobre la vivienda digna en el ámbito local. En este caso, a partir de la instrumentalización de los programas urbanos integrales, que a la par de legalización de la tenencia y a fin de justificar la relocalización de población, incorporaron obras de servicios, infraestructuras y equipamientos en sectores precarizados. Es decir, las grandes líneas de financiamiento internacional tienen injerencia en nuestras ciudades, tal como se promulgaba en la década de 1970 con el auge del neoliberalismo. En este contexto, los PUI instrumentalizados en la ciudad de Santa Fe buscaron mejorar las condiciones de vida de la población a partir de la intervención integral y la recualificación urbana ; legitimando estas obras a partir de operatorias de relocalización, cuya población se localizaba en condiciones precarias de tenencia: sobre los reservorios, la huella del ferrocarril, la traza de la vía, etc. No obstante, los PUI se materializaron a través de convenios que incorporaron, a su vez, organizaciones sociales como médulas de legitimación entre el gobierno y la población, como lo es el MSLT.

En segundo lugar, consideramos ciertas contradicciones entre las premisas promulgadas por el gobierno sobre el prototipo de vivienda universal, y su instrumentalización en los prototipos investigados. En este sentido, comprobamos que estos módulos habitacionales emitidos por aparatos estatales, distan de cumplir los cuatro requisitos pautados de universalidad, flexibilidad de crecimiento, diversidad formal y tipológica, y eficiencia energética.

Por un lado, consideramos contradictorio que todas las familias beneficiarias de planes sociales puedan enmarcarse ideológicamente en una familia tipo, universal, y que estas puedan reproducir sus condiciones de vida en un módulo habitacional de $30 \mathrm{~m} 2$. Tampoco es factible suponer esta afirmación sólo a las familias beneficiarias de planes sociales, dado que estas situaciones no son recomendables para ninguna familia, más allá de la condición social que se encuentre. Por el otro, desde los inicios se proyecta que convivan en una misma habitación varios integrantes del grupo familiar, o todos, dependiendo el caso; así como que también coexistan diversas actividades, incluyendo las de descanso, alimentación, reproducción sexual, recreación, entre otras. Esto implica que los hijos duerman en el mismo local que sus padres, u otras situaciones complejas según la composición del grupo familiar. De modo similar, el prototipo de un dormitorio implica que parte de la familia reproduzca sus condiciones de vida en la misma habitación 
multifunción con todas las actividades antes mencionadas, realizadas en forma simultánea por los diversos integrantes del grupo familiar.

Por otro lado, no encontramos en los prototipos instrumentalizados en la relocalización alguna novedad respecto a la tipología tradicional de vivienda social; y tampoco en relación al uso de energías sustentables. En consonancia, si bien es cierto que el prototipo Al contempla la posibilidad de ampliación sobre la platea, esta ya se encuentra proyectada, y no sobre los fundamentos de la flexibilidad de crecimiento de acuerdo a los requisitos de cada familia. Por ejemplo, no hay mayores posibilidades de expansión de las nuevas habitaciones más que hacia atrás o bien sobre uno de los laterales. Tampoco lo es en cuanto a la construcción sobre la planta alta, dado que esta se ve limitada en ambos prototipos al ser la cubierta de chapa. No obstante, esto sí es posible en los casos en que las familias construyan por fuera del módulo. Por último, los principios de ayuda mutua y esfuerzo compartido convenidos, se ven quebrantados al estar las viviendas construidas por una empresa de premoldeados, donde las placas son montadas por obreros especializados de la propia empresa, cuestión que pudimos comprobar en el trabajo de campo.

En este sentido y por ultimo, buscamos aportar algunos elementos que avancen en esta perspectiva, y que por otro lado, contribuyan a la superación del tratamiento discursivo estigmatizante de la población, en pos de un marco constructivo que avance en el mejoramiento de las condiciones de vida desde el propio núcleo argumental del cual se nutren las políticas urbanas.

\section{BIBLIOGRAFÍA}

Abramo, P. (Org.) 2003. "A Cidade da informalidade. O desafio das cidades latino-americanas". Rio de Janeiro, Editor: Sette Letras.

Calderón Cockburn, J. 2001. "Análisis comparativo de la población beneficiada y la no beneficiada por el Plan Nacional de Formalización”. En "¿Ha mejorado el bienestar de la población? Balance de las principales políticas y programas sociales”. Lima: Instituto Nacional de Estadística e Informática (INEI) - Programa Mecovi.

Cliclevsky, N. 1997. "Regularización dominial: solución para el hábitat "popular" en un contexto de desarrollo sustentable?", en Cuenya, Beatriz y Ana Falú (comp): Reestructuración del Estado y política de vivienda en la Argentina, CEA, Universidad de Buenos Aires, Buenos Aires, pp. 227-245.

Delgadillo, V. 2014. "Urbanismo a la carta: teorías, políticas, programas y otras recetas urbanas para ciudades latinoamericanas." En Cuadernos Metropole, Vol. 16, $\mathrm{N}^{\circ} 31$. Observatorio da Metropole. São Paulo. Pp. 89-111.

Gutiérrez, A. 2007. "POBRE', COMO SIEMPRE...Estrategias de reproducción social en la pobreza", Ferreyra Editor.

Kowarick, L. 2000. "Estudos urbanos" Editora 34. Sao Paulo, SP, Brasil.

Lefebvre, H. 1969. "El derecho a la ciudad." Barcelona: Península.

Leguizamón, Z. (comp.) 2005. "Trabajo y producción de la pobreza en Latinoamérica y El Caribe: estructuras, discursos y actores", -1a ed. - Buenos Aires: CLACSO

Marx, K. 2012 [1872]. "El Capital". Buenos Aires: Siglo XXI Editores.

Mosso, E. 2016. "Reflexiones epistemológicas sobre la marginalidad urbana como proceso acumulativo". En "Actas V ELMECS", ISSN/ISBN 2408-3976. V Encuentro Latinoamericano de Metodología de las Ciencias Sociales (ELMeCS), Noviembre de 2016, Mendoza, Argentina.

--- 2017. “Interpelaciones ideológicas sobre la vivienda. Políticas urbanas de ordenamiento espacial de la población empobrecida en Santa Fe (1985-2017)" Tesis doctoral. FAPyD-UNR.

Murillo, S. (coord.) 2015. "Neoliberalismo y gobiernos de la vida. Diagrama global y despliegues en Argentina y América Latina", 1era. ed.- Biblos, Buenos Aires.

Núñez, A. (coord.) 2016. “Epistemologías del (Des)orden Territorial”. Mar del Plata: EUDEM. [En prensa].

--- 2012. "Miserias de la propiedad: apropiación del espacio, familia y clase social." Mar del Plata: EUDEM.

---- 2012. "Lo que el agua (no) se llevó. Política urbana: poder, violencia e identidades sociales." Buenos Aires: El Colectivo ediciones. 
Núñez, A. y Bedoya, Á. 2011. "Procesos de regularización en Perú, 1971-2008. El caso de Villa El Salvador". Trabajo presentado en la Especialización en Políticas y Mercados de suelo en América latina. Universidad nacional de Colombia (inédito).

Núñez, A. y Roze, J. 2011. "Reflexiones sobre falacias conceptuales y acciones concomitantes en políticas urbanas y sociales en Argentina.", en THEOMAI n 23.

Pradilla, E., 1983. "El problema de la vivienda en América Latina." Quito, Centro de Investigaciones Ciudad.

Pratesi, A. 2004. "Población Pobre objeto de políticas sociales. El caso de Villa Progreso en Resistencia. Chaco". Tesis Doctoral, PPAS-UM. 